Original Research Article

\title{
Monitoring, evaluation and comparison of adverse effect profile of ciprofloxacin and ofloxacin in acute bacterial exacerbation of chronic bronchitis patients attending pulmonology department of tertiary care teaching hospital: a prospective observational study
}

\author{
Sankar K., S. G. S. Rajesh Reddy V.*
}

Department of Pharmacology, Govt. Siddhartha Medical College (GGH-SMC), Vijayawada, Andhra Pradesh, India

Received: 24 January 2018

Accepted: 06 March 2018

\section{*Correspondence to:}

Dr. S. G. S. Rajesh Reddy V., Email: rajesh.vsgs@gmail.com

Copyright: () the author(s), publisher and licensee Medip Academy. This is an openaccess article distributed under the terms of the Creative Commons Attribution NonCommercial License, which permits unrestricted noncommercial use, distribution, and reproduction in any medium, provided the original work is properly cited.

\begin{abstract}
Background: In 2016, FDA recommended that "serious side effects associated with flouroquinolones generally outweigh the benefits for the patients with acute sinusitis, acute bronchitis and uncomplicated UTI". Throughout India the most commonly used flouroquinolones for above conditions are Ciprofloxacin, Ofloxacin, and Levofloxacin. In this scenario, the present study was undertaken to monitor, evaluate and compare the adverse effect profile of Ciprofloxacin and Ofloxacin in acute exacerbation of chronic bronchitis patients.

Methods: Prospective, observational study done in 200 patients of tertiary care teaching hospital. The patients diagnosed with, acute bronchitis treated with Ciprofloxacin 500mg BD or Ofloxacin 400mg BD for 7 days were included in the study. The study duration was 6 months. ADR causality assessment was done with Naranjo algorithm. Statistical test applied was chi- square test.

Results: 63 ADR were reported in Ofloxacin group and 19 ADR in Ciprofloxacin group. Insomnia was most frequent ADR (40\%) in patients treated with Ofloxacin. There is high incidence of ADRs in ofloxacin group. chi square test showed this difference is statistically significant $(p<0.05)$. Insomnia was significantly higher in ofloxacin group $(\mathrm{p}<0.05)$.

Conclusions: In India frequently used and misused antimicrobials are fluoroquinolones. The present study concluded that no serious ADRs but ofloxacin group of patients have shown increased incidence of ADRs associated with CNS especially Insomnia. However, further large scale, multi-centric studies are needed to confirm these results.
\end{abstract}

Keywords: ABECB, Ciprofloxacin, Insomnia, Ofloxacin

\section{INTRODUCTION}

The first quinolone, nalidixic acid, was isolated as a byproduct of the synthesis of chloroquine and made available for the treatment of urinary tract infections. The introduction of fluorinated 4-quinolones, such as ciprofloxacin and moxifloxacin, represents a particularly important therapeutic advance. They have demonstrated comparable efficacy in treatment of CAP and other infections. The increasing prevalence of penicillin resistance bacteria has resulted in reliance on fluoroquinolones and their widespread use globally. ${ }^{1} \mathrm{FQ}$ popularity is enhanced by their relatively broad spectrum activity, approval in most countries for multiple indications, high bioavailability and Long PAE. The fluoroquinolones constitute about $16 \%$ of the world market for antibiotics and it is projected that as the global demand for antibacterial drugs grow, by 2019, fluoroquinolones use would increase. ${ }^{2}$ Consequently, fluoroquinolone- resistant bacteria and reported cases of 
adverse reactions to this drug class would likely increase. Even though, fluoroquinolones have excellent pharmacokinetic properties and are well tolerated by children and adults, there are concerns for their safety. Many adverse drug reactions (ADR) have been reported during clinical trials and post-marketing surveillance. ADRs frequently involve the gastro-intestinal tract (GIT), musculoskeletal system, central nervous system (CNS), dermatological and the hepatic systems. ${ }^{3,4}$ Rare and potentially fatal side effects, however, have resulted in the withdrawal from the U.S. market of lomefloxacin, sparfloxacin (phototoxicity, QTc prolongation), gatifloxacin, temafloxacin, trovafloxacin (hepatotoxicity), grepafloxacin (cardiotoxicity), and clinafloxacin (phototoxicity). In all these cases, the side effects were detected by post marketing surveillance. In 2016, FDA recommended that "serious side effects associated with flouroquinolones generally outweigh the benefits for the patients with acute sinusitis, acute bronchitis and uncomplicated UTI" The FDA further recommended that fluoroquinolone should be reserved for use in patients who have no alternative treatment options for acute bacterial sinusitis (ABS), acute bacterial exacerbation of chronic bronchitis (ABECB), and uncomplicated urinary tract infections (UTIs). Even after FDA warning many clinicians and hospitals throughout India continue to prescribe flouroquinolones for ABECB. In this scenario, the present study was undertaken to asses and compares the adverse effect profile of Ciprofloxacin and Ofloxacin in acute bronchitis patients.

The objective of this study was to monitor, evaluate and compare the adverse effect profile of Ofloxacin and Ciprofloxacin in acute exacerbation of chronic bronchitis.

\section{METHODS}

The study protocol was approved by the Institutional Ethics Committee and the study was conducted in accordance with the Declaration of Helsinki. Prospective, observational study done in 200 patients of clinically diagnosed acute bronchitis in the department of pulmonology, SMC/GGH, Vijayawada. Newly diagnosed 200patients of acute bronchitis (July 2017-December 2017), pulmonology outpatient department, GGH SMC VJA of age 18-65 years of either gender who were prescribed either ciprofloxacin $(n=100)$ ofloxacin $(n=$ 100) and who gave written informed consent were enrolled. One group of the patients received Tab. Ofloxacin $400 \mathrm{mg}$ BD and other group received Tab. Ciprofloxacin $500 \mathrm{mg}$ BD for 7 days. The study duration was 6 months (July 2017- December 2017). Information regarding ADRs were collected from patients by selfreporting by patients, patients were given mobile number of senior residents and postgraduates of departments of pharmacology to report any untoward effects during therapy and they were also given printed forms which consists of expected ADRs from flouroquinolones and asked them to tick ADRs they have experienced during therapy and submit form at end of one week The pattern of
ADRs reported were analyzed. ADR causality assessment was done with Naranjo algorithm.

\section{Inclusion criteria}

- Patients of 18 to 65 years of age of either sex were included in the study.

- Clinically diagnosed patients of acute exacerbation of chronic bronchitis treated with Ofloxacin or Ciprofloxacin.

\section{Exclusion criteria}

- Patients with known hypersensitivity to Flouroquinolones.

- $\quad$ Children, pregnant women and nursing mothers.

- Patients suffering from Diabetes, Myasthenia Gravis, Epilepsy, Psychosis and other CNS disorders.

- $\quad$ Patients suffering from cardiac failure, renal failure and hepatic failure.

\section{Statistical methods}

Data was analyzed using frequency and percentages. Chisquare test is used for comparing attributes and variables of study. $p<0.05$ will be considered as significant. Data was described in form of tables and graphs. Data was entered in Microsoft excel 2007. IBM SPSS software version 21 is used for calculations.

\section{RESULTS}

In Ofloxacin group 63 patients reported ADRs out of which 40 are male and 23 are female. In Ciprofloxacin group, 19 patients reported ADR, of which 10 were men and 9 women (Figure 1).

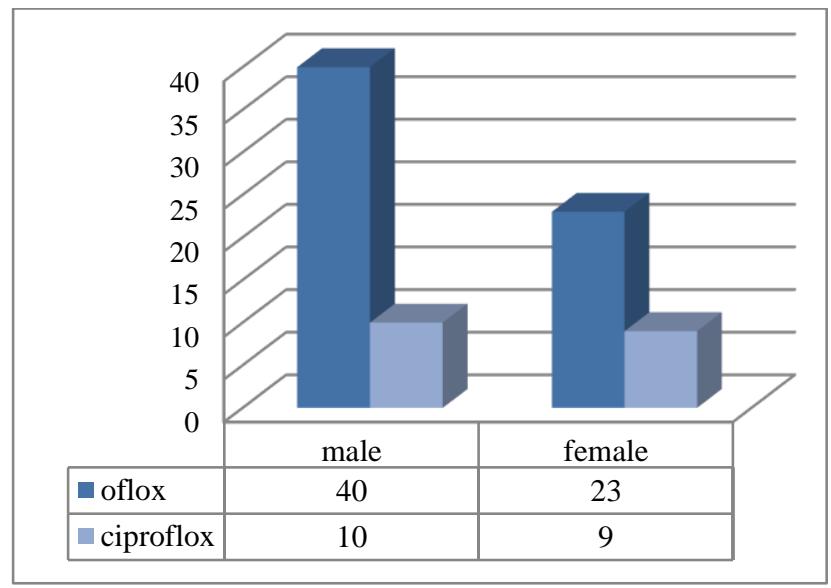

Oflox- Ofloxacin

Ciproflox- Ciprofloxacin

Figure 1: Sex wise distribution of ADR.

Out of 63 ADRs in ofloxacin group 50 patients reported ADRs associated with Central nervous system (CNS), 11 patients reported ADRs associated with Gastrointestinal tract (GIT) and 2 were associated with skin and its 
appendages (Hypersensitivity). Out of 19 ADRs in ciprofloxacin group 10 patients reported ADRs associated with CNS, 08 patients reported ADRs associated with GIT and 01were associated with skin and its appendages (Figure 2).

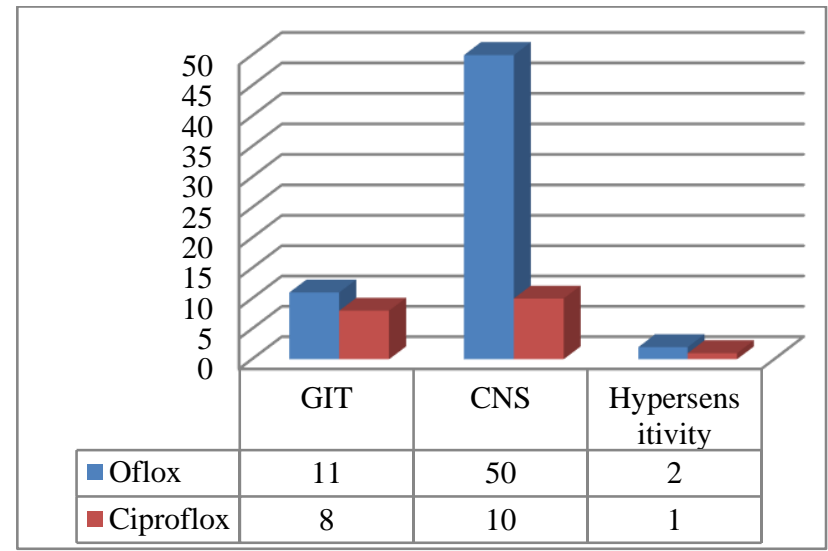

Oflox- Ofloxacin

Ciproflox- Ciprofloxacin

Figure 2: System wise distribution of ADR.

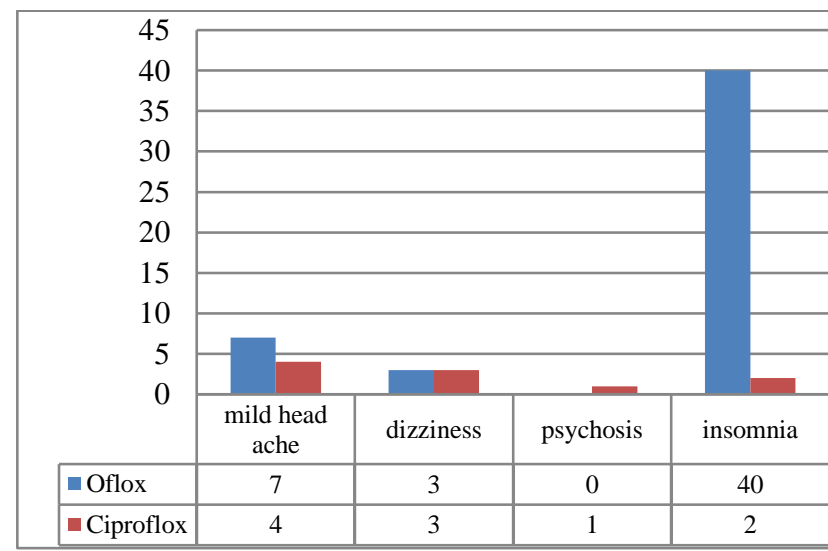

Oflox- Ofloxacin

Ciproflox- Ciprofloxacin

Figure 3: CNS ADRs of Ofloxacin and Ciprofloxacin.

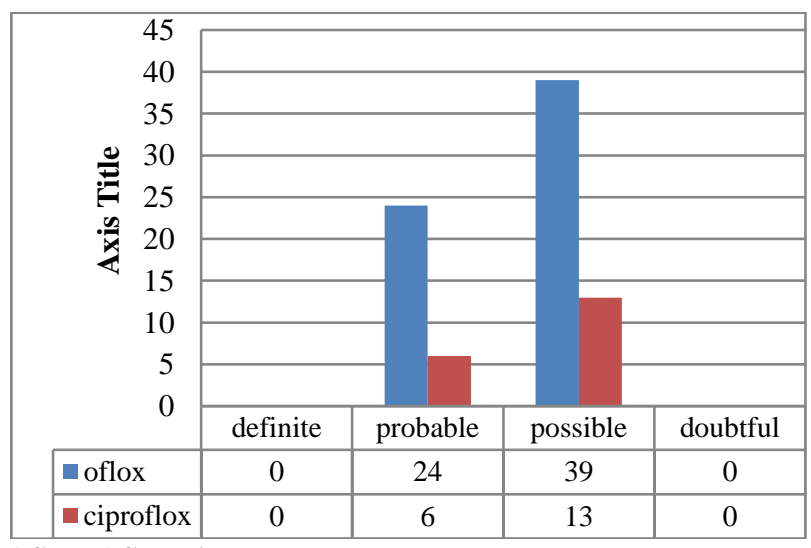

Oflox- Ofloxacin

Ciproflox- Ciprofloxacin

Figure 4: Causality assessment of Ofloxacin and Ciprofloxacin ADRs.
Out of 50 CNS ADRs reported in ofloxacin group 40 patients reported insomnia, 7 patients reported headache and 3 patients reported dizziness. Out of 10 CNS ADRs reported in ciprofloxacin group 2 patients reported insomnia, 4 patients reported headache, 3 patients reported dizziness and 1 case of psychosis was reported (Figure 3 ). Chi square test showed this difference in CNS ADRs is statistically significant $(\mathrm{p}<0.001)$. Insomnia was significantly higher in Ofloxacin group $(\mathrm{p}<0.001)$.

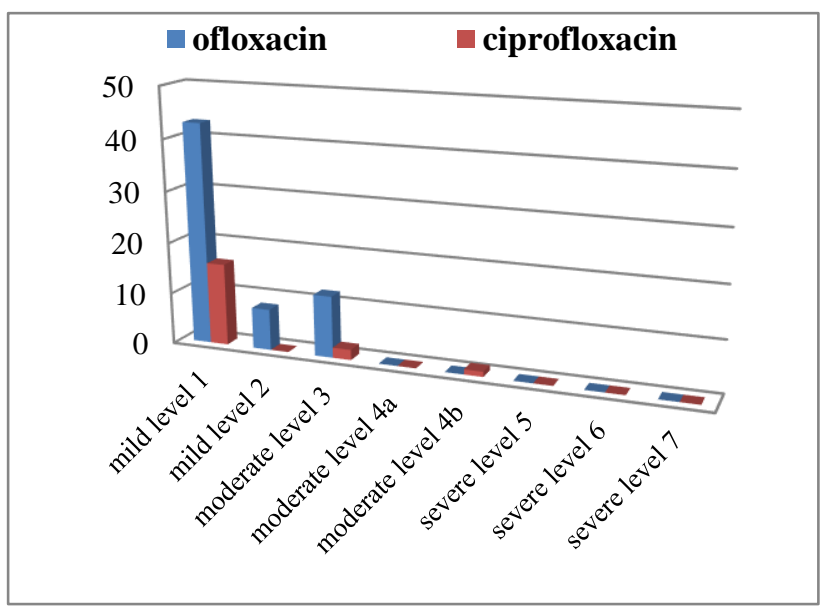

Figure 5: Severity of ADRs.

\section{DISCUSSION}

In general, flouroquinolones are well tolerated, with most side effects being mild to moderate. Common side effects include gastrointestinal effects such as nausea, vomiting and diarrhea, CNS side effects such as headache, dizziness and insomnia. On occasion, serious side effects occur.

In this study ADRs related to neurological disorders especially insomnia, gastrointestinal disorders, and skin and appendages disorders were reported frequently. This trend is similar to the previous ADRs reported to fluoroquinolones. ${ }^{5}$ However, few studies had reported more gastrointestinal than neurological ADRs. ${ }^{6}$ Our findings are contrasting to postmarketing surveillance of ciprofloxacin alone, as well as those of ciprofloxacin, norfloxacin, and ofloxacin showed a preponderance of ADR reporting to skin and appendages, followed by neurological and gastrointestinal disorders.

Previous studies comparing ADRs attributed to fluoroquinolones and other widely used antibiotic classes (cephalosporins, penicillins, and macrolides) reported gastrointestinal disorders, followed by skin and appendages disorders to be more common with other antibiotics. $^{7}$ Another study involving ADRs to all classes of antibiotics reported to a peripheral pharmacovigilance center in India, over a 3-year period, showed that dermatological (47.4\%) and gastrointestinal(39.3\%) ADRs were the most prevalent. 
In few earlier studies insomnia has been reported in about $4.7 \%$ of patients treated with ofloxacin, while psychosis occurs in $<1 \%$ of patients. ${ }^{8,9}$ Therefore, reports of insomnia are expected with quinolones, as well as delirium and psychosis. Insomnia has also been reported to the French database as a common ADR (8\%). In this study insomnia is most frequent ADR, occurred in $40 \%$ patients treated with ofloxacin and in $2 \%$ patients treated with ciprofloxacin.

Floxacin and other flouroquinolones had become one of the most commonly prescribed class of antibiotics. Nearly half of these prescriptions were for conditions not approved by FDA according to study supported in part by agency for health care research and quality. Additionally they are commonly prescribed for medical conditions that are not even bacterial to begin with, such as viral infections, or those to which no proven benefit exists.

The mechanism of toxicity of flouroquinolones have been attributed to their interaction with different receptor complexes, such as blockade of GABAa receptor complex within central nervous system, leading top excitotoxic type effects and oxidative stress. ${ }^{10,11}$

Despite persistent warning signs, the FDA didn't take action until 2008, at which point they added a black box warning about severe tendon damage. They waited another five years before issuing a warning about permanent neuropathy.

\section{CONCLUSION}

In India most frequently used and misused antimicrobials are fluoroquinolones. The present study concluded that no serious ADRs noted in both groups. But ofloxacin group of patients have shown increased incidence of ADRs associated with CNS especially Insomnia. However, further large scale, multi-centric studies are needed to confirm these results and to identify rare serious adverse effects which are not elicited in this study. Inspite of ADR profile of commonly used fluroqinolones in this study is mild to moderate in nature, according to the US FDA (0512-2016) flouroquinolones should be reserved drugs for the treatment patients suffering from acute bronchitis.

\section{ACKNOWLEDGEMENTS}

Authors would like to thank Dr. Niraj Jain, Dr. Alok Pandey, for his support during study.

Funding: No funding sources Conflict of interest: None declared

Ethical approval: The study was approved by the Institutional Ethics Committee of Government Siddhartha Medical College, Vijayawada, Andhra Pradesh, India

\section{REFERENCES}

1. Goldstein EJ, Garabedian-Ruffalo SM. Widespread use of fluoroquinolones versus emerging resistance in pneumococci. Clin Infect Dis. 2002; 35:1505-11.

2. Oreagba IA, Oshikoya KA, Ogar C, Adefwin AO, Ibrahim A, Awodele O, et al. Adverse reactions to fluoroquinolones in the Nigerian population: an audit of reports submitted to the National Pharmacovigilance Centre from 2004 to 2016. Pharmacol Res Perspect. 2017 Apr;5(2):e00297.

3. Tytler BA, Mijinyawa N, Ida A. Comparative quality of fluoroquinolone tablets marketed in some towns in Northern Nigeria. J Pharm Bioresources. 2007;4:8-13.

4. Stahlmann R, Lode HM. Risks associated with the therapeutic use of fluoroquinolones. Expert Opin Drug Saf. 2013;12:497-505.

5. Halkin H. Adverse effects of the fluoroquinolones. RevInfect Dis. 1988; 10: S258-S261.

6. De Sarro A, De Sarro G. Adverse reactions to fluoroquinolones: an overview on mechanistic aspects. Curr Med Chem. 2001;8:371-84.

7. Norrby SR. Side-effects of quinolones: comparisons between quinolones and other antibiotics. Eur J Clin Microbiol Infect Dis. 1991;000:378-83.

8. Fish DN. Fluoroquinolone adverse effects and drug interactions. Pharmacotherapy. 2001;21(10 Pt 2):253$72 \mathrm{~S}$.

9. de Bazignan AD, Thiessard F, Miremont-Salame' G. Psychiatric adverse effects of fluoroquinolones: review of cases from the French pharmacologic surveillance database. Rev Med Interne 2006;27(6):448-52.

10. De Sarro A, Cecchetti V, Fravolini V, Naccari F, Tabarrini, De Sarro G. Effects of novel 6Desfluoroquinolones and Classic Quinolones on pentylenetetrazole-induced Seizures in Mice". Antimicrob Agents Chemother. 1999;43(7):1729-36.

11. Unseld E, Ziegler G, Gemeinhardt A, Janssen U, Klotz U. Possible interaction of Fluroquinolones with the benzodiazepine-GABA A -Receptor complex. July 1990;30(1):63-70.

Cite this article as: Sankar K, Reddy SGSRV. Monitoring, evaluation and comparison of adverse effect profile of ciprofloxacin and ofloxacin in acute bacterial exacerbation of chronic bronchitis patients attending pulmonology department of tertiary care teaching hospital: a prospective observational study. Int J Basic Clin Pharmacol 2018;7:729-32. 